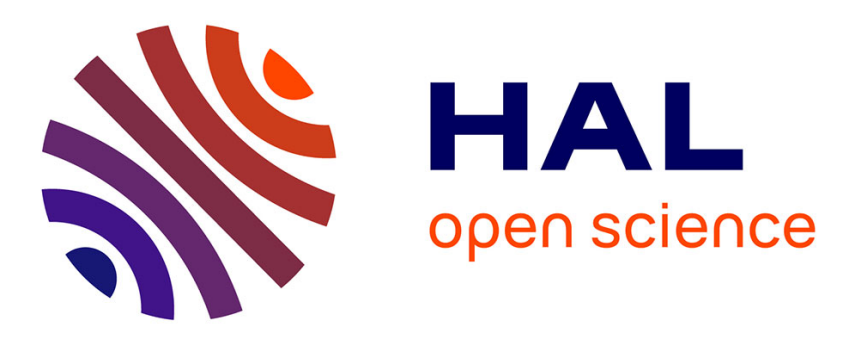

\title{
Exploring Various Orientation Measurement Approaches Applied to a Serious Game System for Functional Rehabilitation
}

\author{
Halim Tannous, Dan Istrate, Aziz Benlarbi-Delai, Julien Sarrazin, Mohamad \\ Idriss, Marie-Christine Ho Ba Tho, Tien Tuan Dao
}

\section{To cite this version:}

Halim Tannous, Dan Istrate, Aziz Benlarbi-Delai, Julien Sarrazin, Mohamad Idriss, et al.. Exploring Various Orientation Measurement Approaches Applied to a Serious Game System for Functional Rehabilitation. EMBC 2016 38th Annual International Conference of the IEEE Engineering in Medicine and Biology Society, Aug 2016, Orlando (FL), United States. hal-01359984

\section{HAL Id: hal-01359984 https://hal.sorbonne-universite.fr/hal-01359984}

Submitted on 5 Sep 2016

HAL is a multi-disciplinary open access archive for the deposit and dissemination of scientific research documents, whether they are published or not. The documents may come from teaching and research institutions in France or abroad, or from public or private research centers.
L'archive ouverte pluridisciplinaire HAL, est destinée au dépôt et à la diffusion de documents scientifiques de niveau recherche, publiés ou non, émanant des établissements d'enseignement et de recherche français ou étrangers, des laboratoires publics ou privés. 


\title{
Exploring Various Orientation Measurement Approaches Applied to a Serious Game System for Functional Rehabilitation
}

\author{
Halim Tannous, Dan Istrate, Aziz Benlarbi-Delai, Julien Sarrazin, Mohamad Idriss, Marie-Christine Ho \\ Ba Tho, Tien Tuan Dao
}

\begin{abstract}
Serious games have been established as a complementary tool for conventional rehabilitation. This trend proved to be beneficial for both experts and patients since the first are able to assign and monitor virtual reality games for their patients to be executed at home, and the latter are greatly motivated by the challenges of these games rather than the repeatable and boring rehabilitation movements. In a previous work, we proposed a serious game for lower limb rehabilitation of the musculoskeletal system using the Microsoft Kinect ${ }^{\mathrm{TM}}$ camera and a virtual environment. However, this system suffers from inaccurate kinematic measurement due to various external sources. Therefore, this paper explores various kinematic measurement technics to improve the accuracy of our system. Six real time orientation measurement algorithms were assessed and compared, using multiple types of sensors. The results obtained showed a better performance for our system. In fact, this study reinforced a clearer view on a low cost, real time portable system to be implemented at home.
\end{abstract}

\section{INTRODUCTION}

Recent years have witnessed a growing interest in the integration of games in several rehabilitation fields, with the main purpose of adding a motivational aspect for this procedure, while allowing the experts to easily monitor their patients. Furthermore, numerous visual and portable sensors have been employed in these studies. The most commonly used sensor is the Microsoft Kinect ${ }^{\mathrm{TM}}$ [1] due to its low price and its already successful legacy with Xbox games. Chang et al. [2] proved, in their pilot study that using a Kinect-based system can help motivate patients and increase the rehabilitation's efficiency. In their work, they showed that the number of correctly executed movements during the use of their system increased drastically, compared to a baseline without its use. Zannatha et al. [3] presented a serious game for upper limb rehabilitation using the Kinect $^{\mathrm{TM}}$ and Electromyography (EMG) sensors attached to the arm. The system used an inverse kinematic algorithm to determine the angles of the upper segments and studied the EMG signals generated while practicing the exercises. However they did not study the accuracy of their angle measurements, or the responses of actual test subjects, but instead, they studied only the EMG signals. Lozano-Quilis et al. [4] developed a virtual rehabilitation game for multiple sclerosis called RemoviEM, using a natural user interface and showed improvements from patients who tested their system. Commercially available serious games are also present

H. Tannous, D. Istrate, M. Idriss, MC. Ho Ba Tho, TT. Dao are with Sorbonne University, Université de technologie de Compiègne, CNRS, UMR 7338 Biomechanics and Bioengineering (BMBI), Centre de recherche Royallieu - CS 60319 - 60203 Compiègne cedex (halim.tannous@utc.fr).

A. Benlarbi-Delai and J. Sarrazin are with Sorbonne University, Université Pierre et Marie Curie, L2E, Place Jussieu, Paris. nowadays like Jintronix [5] rehabilitation software, which consists of a home based rehabilitation system with expert monitoring.

Other researchers focused on inertial and magnetic sensors, since the Kinect ${ }^{\mathrm{TM}}$ suffers from several setbacks caused mainly by the superposition of body parts while performing exercises, and also by the changes in the lighting of the scene. Cho et al. [6] used a connected cylinder shaped object in order to implement a proprioception rehabilitation game for stroke patients. But, despite their positive results, they concluded that the patients became accustomed to the game and were able to perform the task easily. Paraskevopoulos et al. [7] set some guidelines for designing games for Parkinson's disease patients and used the Wiimote $^{\mathrm{TM}}$ to design two games. The system evaluation was done using questionnaires and participant's suggestions and showed an increase in motivation. Chen et al. [8] used a force plate to implement an interactive serious game between patient and expert. The results showed a great improvement for participants who used the system compared to a control group.

In order to track the movement of the person, and offer the expert good and accurate data from each session, several orientation estimation techniques have been studied using various, commercial and developed, inertial motion units (IMU) or magnetic, angular rate and gravity sensors (MARG). Wireless body area networks have been largely studied as a tool for motion capture, in particular for orientation estimation and measurement. One of the first successful attempts of implementing a joint angle measurement using IMU was done by Williamson et al. [9], who compared the accelerometer's and gyroscope's ability to measure joint angles and angular rates, then compared the results to the traditional goniometer. The gyroscope proved to be more efficient than the accelerometer in both types of measurements. Myagoitia et al. [10] found great results when comparing angles and angular velocities calculated using gyroscopes, and linear and angular acceleration calculated using accelerometers with a reference motion capture system [11]. Then, in 2005 and 2006 Dejnabadi et al. [12] successfully coupled the calculation of the hip, knee, ankle [13] angles with a personalized biomechanical reference based on each patient. The study's results were closer to reality than those calculated without coupling. Takeda et al. [14] used several accelerometers placed on the lower part of the body to try and calculate the position of each joint and the angles between them, starting from the bottom and moving upwards. Unfortunately, the results were not significant since their system was too complex. More accurate commercial sensors were developed during later years as Perez et al. [15] 
implemented an angle measurement system of the upper body using available quaternion based sensors. Many other contributions have been made in this field of study, and many more are needed to perfect this technology, both hardware and software wise. These systems have lots of advantages compared to visual sensors, but also present some challenges [16].

In this paper we focus on the improvement of kinematic measurement for our rehabilitation system presented in [17]. In fact, we performed a comparison between different algorithms tested for orientation measurement, and a new fusion algorithm between the Kinect $^{\mathrm{TM}}$ and Shimmer ${ }^{\mathrm{TM}}$ portable sensors [18]. We note that previous attempts to fuse these two sensors were studied by Feng et al. [19] in order to obtain a more precise position for the 3D joints obtained from the visual sensor. There were also attempts by Destelle et al. [20] who used the Kinect ${ }^{\mathrm{TM}}$ to get the initial position, and then calculated the orientations using inertial sensors. However, and to our knowledge, there is no study of quaternion orientation fusion between visual and inertial sensors for body tracking.

Finally, we discuss the coupling of these kinematic data with a multi-physical musculoskeletal system that could allow the expert to access more useful information in order to assess the patient's condition.

\section{MATERIALS AND METHODS}

\section{A. Serious Game}

The main focus of our work is to implement a real time, enjoyable home based rehabilitation system for functional rehabilitation, something we began presenting in our previous work [17], where we developed a series of exergames executed by patients using the Kinect $^{\mathrm{TM}}$ sensor. The developed exergames successfully motivated the patients, but a more realistic game-like environment was needed to try and maximize the amusement of the subjects. Therefore, we developed a couple of new real life scenarios; the first is a soccer scene where the patient needs to shoot the ball when the moving crosshair is in front of a cone in order to hit it. The velocity of contact between the foot and the ball, as well as the point of the contact were used to simulate the trajectory of the ball, the level of difficulty varies based on the speed of the crosshair and the dimensions of the cones (Figure 1).

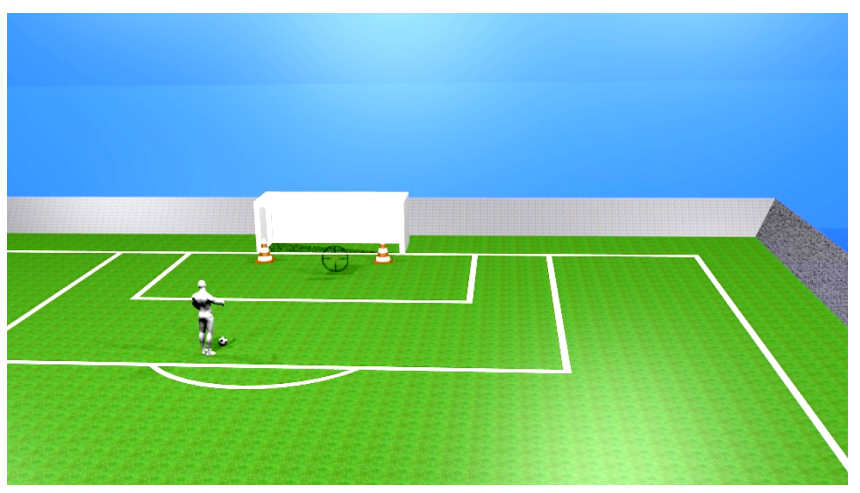

Figure 1. Soccer Scene Example

Another game that was developed is based on gait training, where the subject needs to collect appearing objects in a scene and place them on a table.
As stated previously, the Kinect ${ }^{\mathrm{TM}}$ was able to assess all of the previously developed exergames, but a more precise orientation and angle measurement system was needed to compensate the loss of information due to the occlusion of body parts, and to the limited accuracy of visual motion capture. In this sense, and since the game is meant for lower limb rehabilitation, we decided to use the Kinect ${ }^{\mathrm{TM}}$ sensor as a tool to plot the position of the body in the 3D virtual environment, and calculate the orientation and angles of the upper limbs, then couple this system with a wireless sensor body area network (WBAN), using commercially available Shimmer ${ }^{\mathrm{TM}}$ IMUs to get a more accurate reading on lower limbs. The IMU architecture is shown in Figure 2.

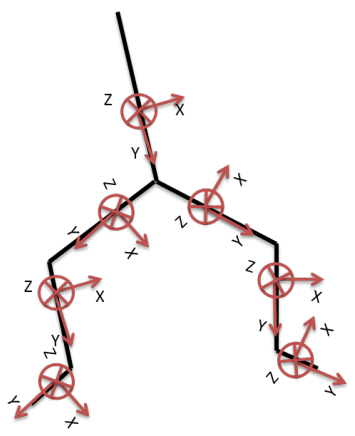

Figure 2. Shimmer sensors architecture

Using this new visual and inertial sensor architecture, the expert will be able to receive an accurate data reading of the knee, thigh and shank angles, and a more precise reading on the bio-feedbacks of the human musculoskeletal system during the rehabilitation exercises.

The next step was to evaluate the different algorithms for lower limb orientation measurement using IMUs and study an implementation of an extended Kalman filter fusion between the Kinect ${ }^{\mathrm{TM}}$ and Shimmer ${ }^{\mathrm{TM}}$ sensors for improving the accuracy of the measurements.

\section{B. Evaluation of Existing Orientation Measurement Algorithms}

A series of algorithms were studied for lower limb angle measurement, using both visual and inertial measurement technics. The measurement process was performed using the Kinect $^{\mathrm{TM}}$ sensor and 2 attached Shimmer ${ }^{\mathrm{TM}}$ sensors on the thigh and shank respectively, in order to measure the knee angle. In fact, five algorithms were tested: (1) 3D accelerometers [9], (2) Kalman filter between 3D accelerometers and gyroscopes [21], (3) Quaternion based extended Kalman filter using 3D accelerometers, gyroscopes and magnetometers [22], (4) Quaternion based Gradient Descent algorithm [23], and Quaternion based Kinect $^{\mathrm{TM}}$ algorithm.

\section{Multisensory fusion}

A multi-sensor fusion scheme was developed using Kinect $^{\mathrm{TM}}$ and Shimmer ${ }^{\mathrm{TM}}$ sensors. Our approach consists of an extended Kalman filter fusion between the quaternions obtained from the Gradient Descent algorithm, which proved to be the best algorithm for orientation estimation using inertial sensors, and the Kinect ${ }^{\mathrm{TM}}$ algorithm. Each quaternion was treated as an input to a separate filter in order to determine the posteriori covariance matrices $\mathrm{P}_{1}$ and $\mathrm{P}_{2}$ for the 
two sources. The two sources are then fused together using equations (1) and (2).

$$
\begin{gathered}
\mathrm{P}=\mathrm{P}_{1}^{-1}+\mathrm{P}_{2}^{-1} \\
\mathrm{X}=\mathrm{P}^{-1}\left(\mathrm{P}_{1}^{-1} \mathrm{X}_{1}+\mathrm{P}_{2}^{-1} \mathrm{X}_{2}\right)
\end{gathered}
$$

where $\mathrm{X}$ is the output state of the fusion filter and $\mathrm{X}_{1}, \mathrm{X}_{2}$ are the output states of the first and second extended Kalman filters respectively.

Equations (3) and (4) show the evolution model and state vector respectively.

$$
\begin{gathered}
\dot{\mathrm{s}}=\frac{1}{2} \omega * \mathrm{~s} \\
\mathrm{X}=\left[\mathrm{s}_{0} \mathrm{~s}_{1} \mathrm{~s}_{2} \mathrm{~s}_{3} \omega_{\mathrm{x}} \omega_{\mathrm{y}} \omega_{\mathrm{z}}\right]^{\mathrm{T}}
\end{gathered}
$$

Where $\mathrm{s}$ is the quaternion composed of $\mathrm{s}_{0}, \mathrm{~s}_{1}, \mathrm{~s}_{2}$ and $\mathrm{s}_{3}, \omega$ is a matrix containing the angular velocities $\omega_{x}, \omega_{y}$, and $\omega_{z}$ with respect to axis $\mathrm{x}, \mathrm{y}$ and $\mathrm{z}$.

Finally, the covariance matrices of measurement noise $\mathrm{R}_{1}$ and $R_{2}$ were projected from existing values in the literature that measured the error between the Kinect's measured angle and a reference measurement system [24], and the values between the Gradient Descent algorithm and a reference measurement system [23].

\section{RESULTS AND DISCUSSION}

\section{A. Evaluation of Existing Orientation Measurement Algorithms}

The results of the evaluation of different orientation measurement algorithms are shown in Figure 3, Figure 4, and Table 1 respectively.

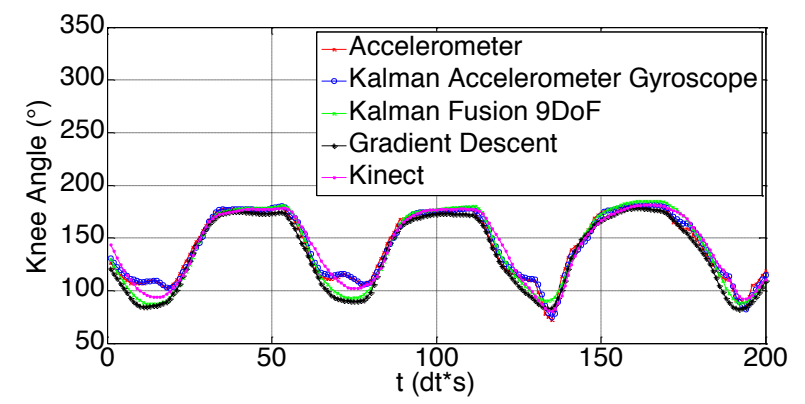

Figure 3. Evaluation of different orientation measurement algorithms through knee angle measurement

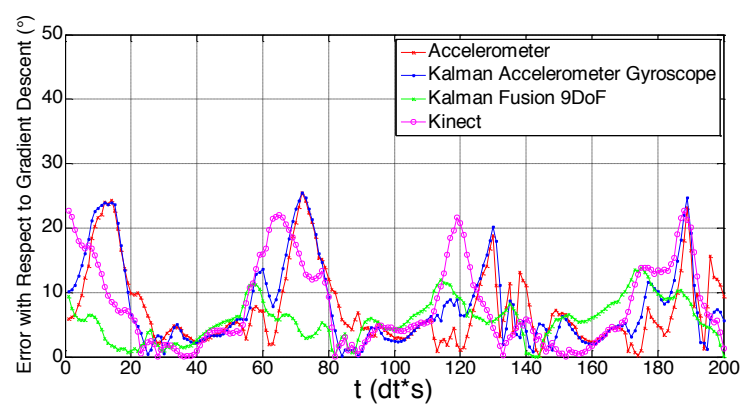

Figure 4. Error computation with respect to Gradient Descent
TABLE I. COMPARISON BETWEEN DIFFERENT ALGORITHMS WITH RESPECT TO GRADIENT DESCENT

\begin{tabular}{|c|c|c|c|c|}
\hline \multirow{2}{*}{ Evaluation } & \multicolumn{4}{|c|}{ Algorithm } \\
\cline { 2 - 5 } & Acc & $\begin{array}{c}\text { Kalman Acc }+ \\
\text { Gyro }\end{array}$ & $\begin{array}{c}\text { Kalman } \\
\text { Fusion } \\
\text { 9DoF }\end{array}$ & Kinect \\
\hline RMS & 12.1936 & 12.5524 & 6.1304 & 10.1837 \\
\hline Correlation & 0.9673 & 0.9713 & 0.9941 & 0.9724 \\
\hline
\end{tabular}

The IMU based algorithms present a more accurate measurement than that of the Kinect ${ }^{\mathrm{TM}}$ since they do not attempt to calculate angles using a visual technic. Furthermore, Madgwick et al. [23] described their gradient descent algorithm and indicated that it has better precision than the quaternion based extended Kalman 9 degrees of freedom (DoF) estimation. Based on these facts, we took the quaternion based algorithm as a reference to which we compared the other methods. These results indicated great correlation between all of the existing algorithms $(>0.96)$ but also a great amount of error in three among them. The Table 1 allows us to eliminate the first and second algorithms based on their results compared to the more complex extended Kalman 9 DoF and Gradient Descent algorithms. Furthermore, the computed RMS between the extended Kalman 9 DoF and Gradient Descent methods in [23] proved to be compatible with our results if extended to two sensors and the existing angle between them. Finally, the large RMS of the Kinect ${ }^{\mathrm{TM}}$ compared to the most effective IMU algorithm proves the need to upgrade our system and combine visual and inertial sensors.

Since the Kinect ${ }^{\mathrm{TM}}$ is a vital part of our game, that allows the positioning of the subject in the virtual environment, and since the IMUs are needed to increase the precision of the measures, the next step of our work focused on developing a fusion protocol between the two systems and implementing it on the lower body.

\section{B. Multisensory fusion}

The results of our fusion algorithm according between the visual and inertial sensor algorithms (Gradient Descent, Kinect) are presented in Figure 5.

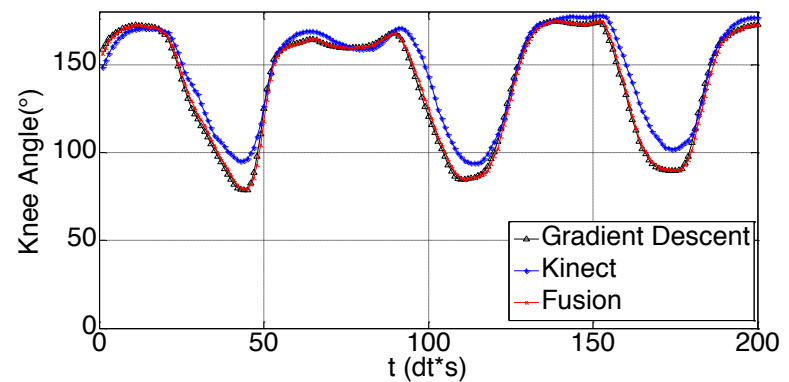

Figure 5. Comparison between Gradient Descent, Kinect and Multisensory fusion algorithm

These results are in agreement with the fact that the values of the angle estimation error of the IMU are significantly less than those of the Kinect ${ }^{\mathrm{TM}}$, therefore the angle deduced from the fused system follows the Gradient Descent quaternion more than the Kinect ${ }^{\mathrm{TM}}$ algorithm, but take into consideration the Kinect's quaternion when the difference is 
significant. The next step in our research will be to evaluate this fusion algorithm with respect to a golden standard reference, e.g. VICON 3D motion capture system, and then fine tune the parameters in order to better estimate the angles using both visual and inertial sensors, to finally obtain a better result from the fusion algorithm.

\section{CONCLUSION}

In this paper, we presented the recent advancements in our serious game for musculoskeletal rehabilitation. We also presented a multisensory fusion method to combine orientations from visual and inertial sensors, that will be compared to a golden reference during the next step. The ultimate goal of this research will be to present the patient with fun and challenging games, while giving the expert accurate and precise data of his patient's lower limbs conditions (angles, bio-feedback ...) through the use of a patient personalized multi-physical musculoskeletal system [25].

\section{ACKNOWLEDGMENT}

This work was funded within the framework of EBIOMED Chair - IUIS (Institut Universitaire d'Ingénierie en Santé). This work was carried out in the framework of the Labex MS2T, which is funded by the French Government.

\section{REFERENCES}

[1] www.xbox.com [last accessed: March 2016].

[2] Chang, Y.-J., Chen, S.-F., \& Huang, J.-D. (2011). A Kinect-based system for physical rehabilitation: a pilot study for young adults with motor disabilities. Research in Developmental Disabilities, 32(6), 2566-70. http://doi.org/10.1016/j.ridd.2011.07.002.

[3] Ibarra Zannatha, J. M., Tamayo, A. J. M., Sánchez, A. D. G., Delgado, J. E. L., Cheu, L. E. R., \& Arévalo, W. A. S. (2013). Development of a system based on 3D vision, interactive virtual environments, ergonometric signals and a humanoid for stroke rehabilitation. Computer Methods and Programs in Biomedicine, 112(2), 239-49. http://doi.org/10.1016/j.cmpb.2013.04.021.

[4] Lozano-Quilis, J.-A., Gil-Gómez, H., Gil-Gómez, J.-A., Albiol-Pérez, S., Palacios-Navarro, G., Fardoun, H. M., \& Mashat, A. S. (2014). Virtual Rehabilitation for Multiple Sclerosis Using a Kinect-Based System: Randomized Controlled Trial. JMIR Serious Games, 2(2), e12. http://doi.org/10.2196/games.2933.

[5] www.jintronix.com [Last accessed: March 2016].

[6] Cho, S., Ku, J., Cho, Y. K., Kim, I. Y., Kang, Y. J., Jang, D. P., \& Kim, S. I. (2014). Development of virtual reality proprioceptive rehabilitation system for stroke patients. Computer Methods and Programs in Biomedicine, 113(1), 258-65. http://doi.org/10.1016/j.cmpb.2013.09.006.

[7] Paraskevopoulos, I. T., Tsekleves, E., Craig, C., Whyatt, C., \& Cosmas, J. (2014). Design guidelines for developing customised serious games for Parkinson's Disease rehabilitation using bespoke game sensors. Entertainment Computing, 5(4), 413-424. http://doi.org/10.1016/j.entcom.2014.10.006.

[8] Chen, P.-Y., Wei, S.-H., Hsieh, W.-L., Cheen, J.-R., Chen, L.-K., \& Kao, C.-L. (2012). Lower limb power rehabilitation (LLPR) using interactive video game for improvement of balance function in older people. Archives of Gerontology and Geriatrics, 55(3), 677-82. http://doi.org/10.1016/j.archger.2012.05.012.

[9] Williamson, R., \& Andrews, B. J. (2001). Detecting absolute human knee angle and angular velocity using accelerometers and rate gyroscopes. Medical \& Biological Engineering \& Computing, 39(3), 294-302. http://doi.org/10.1007/BF02345283.

[10] www.vicon.com [Last accessed: March 2016].
[11] Mayagoitia, R. E., Nene, A. V., \& Veltink, P. H. (2002). Accelerometer and rate gyroscope measurement of kinematics: an inexpensive alternative to optical motion analysis systems. Journal of Biomechanics, 35(4), 537-542. http://doi.org/10.1016/S00219290(01)00231-7.

[12] Dejnabadi, H., Jolles, B. M., \& Aminian, K. (2005). A new approach to accurate measurement of uniaxial joint angles based on a combination of accelerometers and gyroscopes. IEEE Transactions on Bio-Medical Engineering, 52(8), 1478-84. http://doi.org/10.1109/TBME.2005.851475.

[13] Dejnabadi, H., Jolles, B. M., Casanova, E., Fua, P., \& Aminian, K. (2006). Estimation and visualization of sagittal kinematics of lower limbs orientation using body-fixed sensors. IEEE Transactions on Bio-Medical $\quad$ Engineering, 53(7), 1385-93. http://doi.org/10.1109/TBME.2006.873678.

[14] Takeda, R., Tadano, S., Todoh, M., Morikawa, M., Nakayasu, M., \& Yoshinari, S. (2009). Gait analysis using gravitational acceleration measured by wearable sensors. Journal of Biomechanics, 42(3), 22333. http://doi.org/10.1016/j.jbiomech.2008.10.027.

[15] Pérez, R., Costa, Ú., Torrent, M., Solana, J., Opisso, E., Cáceres, C., Gómez, E. J. (2010). Upper limb portable motion analysis system based on inertial technology for neurorehabilitation purposes. Sensors (Basel, $\quad$ Switzerland), 10(12), 10733-51. http://doi.org/10.3390/s101210733.

[16] Hadjidj, A., Souil, M., Bouabdallah, A., Challal, Y., \& Owen, H. (2013). Journal of Network and Computer Applications Wireless sensor networks for rehabilitation applications: Challenges and opportunities, 36, 1-15.

[17] Tannous, H., Dao, T. T., Istrate, D., \& Ho Ba Tho, M. C. (2015). Serious game for functional rehabilitation. 2015 International Conference on Advances in Biomedical Engineering (ICABME), 242-245. http://doi.org/10.1109/ICABME.2015.7323297.

[18] www.shimmersensing.com [Last accessed: March 2016].

[19] Murray-Smith, R. (2014). Fusing Kinect Sensor and Inertial Sensors with Multi-rate Kalman Filter. IET Conference on Data Fusion \& Target Tracking 2014: Algorithms and Applications, 2.3-2.3. http://doi.org/10.1049/cp.2014.0527.

[20] Ahmadi, A., Connor, N. E. O., \& Moran, K. Low-Cost Accurate Skeleton Tracking Based on Fusion of Kinect and Wearable Inertial Sensors. Franc Insight Centre for Data Analytics Dublin City University Dublin, Ireland Anargyros Chatzitofis, Dimitrios Zarpalas , Petros Daras Information Technologies Institute Centre for Research and Technology Hellas Greece, 371-375.

[21] Abyarjoo, F., Barreto, A., Cofino, J., \& Ortega, F. R. Implementing a Sensor Fusion Algorithm for 3D Orientation Detection with Inertial / Magnetic Sensors.

[22] Marins, J. L., Bachmann, E. R., McGhee, R. B., \& Zyda, M. J. (2003). An extended Kalman filter for quaternion-based orientation estimation using MARG sensors. Proceedings 2001 IEEE/RSJ International Conference on Intelligent Robots and Systems. Expanding the Societal Role of Robotics in the the Next Millennium (Cat. No.01CH37180), 4, 2003-2011. http://doi.org/10.1109/IROS.2001.976367.

[23] Madgwick, S. O. H., Harrison, A. J. L., \& Vaidyanathan, A. (2011). Estimation of IMU and MARG orientation using a gradient descent algorithm. IEEE ... International Conference on Rehabilitation Robotics: $\quad$ [proceedings], $2011, \quad 5975346$. http://doi.org/10.1109/ICORR.2011.5975346.

[24] Plantard, P., Auvinet, E., Pierres, A.-S. Le, \& Multon, F. (2015). Pose estimation with a Kinect for ergonomic studies: evaluation of the accuracy using a virtual mannequin. Sensors (Basel, Switzerland), 15(1), 1785-803. http://doi.org/10.3390/s150101785.

[25] Dao, T. T., Tannous, H., Pouletaut, P., Gamet, D., Istrate, D., \& Ho Ba Tho, M. C. (2016). Interactive and Connected Rehabilitation Systems for E-Health. Irbm, 1, 1-8. http://doi.org/10.1016/j.irbm.2016.02.003. 